Stereotactic and Functional

Neurosurgery
Stereotact Funct Neurosurg 2011;89:42-47

DOI: $\underline{10.1159 / 000321186}$
Received: April 7, 2010

Accepted after revision: September 12, 2010

Published online: December 23, 2010

\title{
Cortical Areas Involved in Numerical Processing: An Intraoperative Electrostimulation Study
}

\author{
Song $\mathrm{Pu}^{\mathrm{a}}$ Yong-nian $\mathrm{Li}^{\mathrm{b}}$ Chen-xing $\mathrm{Wu}^{\mathrm{c}}$ Yong-zhi Wang ${ }^{\mathrm{c}}$ Xin-lin Zhou ${ }^{\mathrm{b}}$ \\ Tao Jiang ${ }^{c}$ \\ a Department of Neurosurgery, The First Affiliated Hospital of Harbin Medical University, Harbin, \\ ${ }^{b}$ State Key Laboratory of Cognitive Neuroscience and Learning, Beijing Normal University, and \\ 'Department of Neurosurgery, Beijing Tiantan Hospital Affiliated to Capital Medical University, Beijing, China
}

\section{Key Words}

Awake craniotomy $\cdot$ Gliomas $\cdot$ Surgical treatment $\cdot$

Electrical stimulations

\begin{abstract}
Background: Numerical processing is important in our everyday lives. However, very few attempts have been made to map the numerical processing function areas during lesion surgery. Objective: To identify and protect the cortical areas involved in numerical processing, the authors used the intraoperative brain mapping approach to study numerical processing areas in patients with parietal lobe tumors. Methods: During resection in patients with parietal lobe tumors, local anesthesia was administered and numerical processing mapping was performed. Our mapping procedures were conducted before glioma removal and included somatosensory, language and numerical processing tasks. We focused on the numerical processing task. Results: Different brain sites within the parietal lobe were detected to be specifically related to multiplication or subtraction processing. They displayed precise spatial distribution and overlapped with each other. No brain sites were found to be specifically related to numerical processing in the right hemisphere. Conclusions: To improve the quality of resection while minimizing the neurological deficits, functional boundaries of
\end{abstract}

numerical processing areas should be considered during the removal of a parietal low-grade glioma. Moreover, only the left intraparietal sulcus is necessary for numerical processing, whereas the right intraparietal sulcus does not appear to be critically involved in numerical processing.

Copyright $\odot 2010$ S. Karger AG, Basel

\section{Introduction}

Numerical processing is one of the most important and basic functions of the human brain in our daily lives. Its impairment can cause social and professional disturbances [1]. Nowadays, neuroimaging and neuropsychological studies indicate that the parietal lobe, especially the intraparietal sulcus (IPS), plays an important role in processing and representing numerical magnitude [2-4].

The regular application of the direct stimulation method, that is to identify the eloquent areas when the patient is awake, has recently been confirmed to improve the postoperative function [5-8]. However, very few attempts were specifically made to map the numerical processing function areas during lesion surgery $[9,10]$. To identify and protect cortical areas involved in the numerical processing function, we used the intraoperative brain mapping approach to study numerical processing in pa-

Dr. Tao Jiang

Department of Neurosurgery

Beijing Tiantan Hospital Affiliated to Capital Medical University

Beijing 100050 (China)

Tel. +86 106709 8039, Fax +861069036038, E-Mail jiangtao369@sohu.com 
tients with brain tumors. The lesions were located close to brain regions that may be involved in mathematical functions.

The present study focuses on the numerical processing functions of the brain, especially the subtraction and multiplication tasks. At the same time, the recent progress made in direct electrostimulation methods is taken into account. The cortical organization of areas involved in subtraction and multiplication tasks can be detected and analyzed using the surgical data from intraoperative brain mapping.

\section{Methods}

\section{Case Recruitment}

Between May 2008 and January 2009, brain mapping for the numerical processing task was offered to 5 patients ( 3 men and 2 women) with various low-grade gliomas (LGGs). All cases were identified as right-handed when assessed by a Chinese version of the Edinburgh Handedness Inventory [11-13]. When a lesion was close to the inferior parietal lobe, a numerical processing task was added to the standard brain mapping. This region was presumed to be involved in numerical processing $[1,9,10]$.

All patients and their families signed written informed consent forms to undergo direct brain mapping.

\section{Preoperative and Postoperative Evaluation}

The preoperative neurological clinical test results were normal for all patients. The language ability was tested by a speech therapist using a Chinese version of the West Aphasia Battery [11-13]. The Mini-Mental State Examination was used as a preoperative neuropsychological test. The Karnofsky Performances Scale score was evaluated for all patients. All patients underwent preoperative numerical processing examinations by a neurophysiologist team to rule out any specific deficits. Numerical processing, including number comparison (which of 2 numbers presented in Arabic digits is larger), single-digit multiplication and simple subtraction, was performed using a computer system with a liquid crystal display screen $[9,10]$. The duration of each stimulation was $4 \mathrm{~s}$.

\section{Preoperative Training}

Within 3 days prior to the surgery, patients were informed in detail about the monitoring procedure of the intraoperative stimulation. Patients were familiarized with the stimulus material and trained to perform the naming and calculating tasks. The task was based on the individual capabilities of each patient. If a patient could not name certain objects, these pictures were removed from the stimulus pool.

\section{Intraoperative Cortical Stimulation}

Language, motor and sensorimotor functions were mapped first [5-8]. Then, in order to investigate the function of IPS in arithmetic processing, subtraction and multiplication tasks (4-6 $\mathrm{mA}$ ) were carried out $[9,10]$. One subtraction and 1 multiplication block were presented for all sites exposed to the bone flap. Num- bers were printed on sterilized paper and placed on the cortical surface. Moreover, a photograph was obtained immediately after the stimulation in order to identify sites of positive stimulations later on.

The subtraction task included 36 subtraction equations (2-digit integer minus 1 -digit integer) from $11-2=9$ to $18-9=9$. The answer for each equation was a single-digit number. Each complete equation consisted of 4 digits. Stimuli were presented at the center of the screen, but the ' $=$ ' sign and the answers were not presented (such as, $11-2$ or $18-9$ ), and the participants had to orally report the answer within $4 \mathrm{~s}$. We also used single-digit multiplication with at least 1 operand $>6$ (e.g. $4 \times 7,3 \times 5,2 \times 8$ ).

Each stimulation site was tested with 2 stimuli. For the first stimulus, we did not use electrostimulation (to check if the patient was able to solve the problem without any stimulation, especially when the previous stimulation had induced a disruption of numerical processing), but for the second stimulus electrostimulation was applied.

Multiplication and subtraction questions were alternated 3 times. If an error occurred, at least 1 stimulus presentation was performed without stimulation to ensure that the patient was able to perform the task correctly [9]. The patients were never informed when the brain was stimulated. The same cortical site was never stimulated successively twice to avoid seizures.

\section{Surgical Strategy}

The surgical approach for the lesion was determined by $\mathrm{T}_{1^{-}}$ and $\mathrm{T}_{2}$-weighted MRI data, supported by information from intraoperative ultrasonography. Tumor removal was conducted using these functional landmarks as boundaries of the resection. The cortical incision procedure was customized according to the results of the cortical mapping procedure [11].

\section{Postoperative Course}

The extent of tumor residue was evaluated by postoperative imaging $3 \mathrm{~h}$ after the surgical procedure (CT scans and MRI), according to the classification method reported by Xie et al. [12]. Conventional MRI and functional MRI were performed in all cases 3 months after surgery. Formal neurological examinations were preoperatively performed once (1-3 days before the operation) and postoperatively twice (within 21 days and 6-12 months after surgery). Numerical processing was also assessed using a computer system.

\section{Results}

Among the 5 patients who underwent numerical processing cortical stimulation, 4 were treated in the left hemisphere and 1 in the right hemisphere.

\section{Intraoperative Stimulation}

Direct stimulation mapping was simple and well tolerable in all patients. Focal seizures during the intraoperative stimulation mapping procedure occurred in 1 case. The focal seizures were controlled by irrigating the cortex with cold saline solution. Twenty-two number-specific 

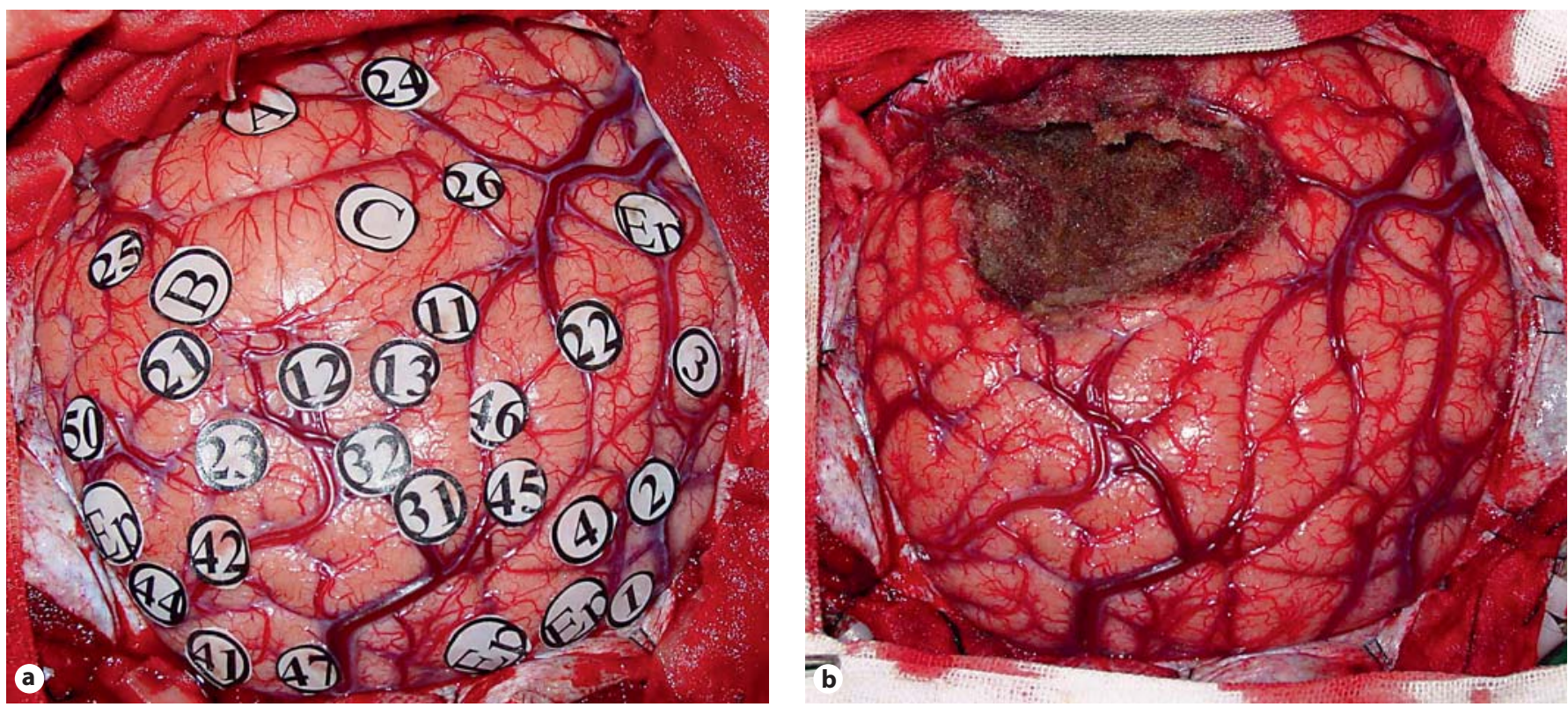

Fig. 1. a Intraoperative cortical mapping before resection of patient 2 . Tumor edge $=\mathrm{A}-\mathrm{C}$; motor areas $=1-4$; counting $=11,12$; naming $=21,23,25$; reading $=31$, 32, 45; multiplication $=41,42,44,46$, 47; subtraction $=41$, 44, 47, 50; multiplication/subtraction $=41,44,47$. $\mathbf{b}$ Cortical mapping after resection of patient 2.

Table 1. The clinical, radiological and pathological summary of patients

\begin{tabular}{|c|c|c|c|c|c|c|c|c|}
\hline Patient No. & Age/sex & $\mathrm{L} / \mathrm{R}$ & Site & Pathology & M & MS & S & Naming \\
\hline 1 & $27 / F$ & $\mathrm{~L}$ & parietal & astrocytoma & no & no & 4 & 1 \\
\hline 2 & $33 / \mathrm{F}$ & $\mathrm{L}$ & $\begin{array}{l}\text { temporal } \\
\text { parietal }\end{array}$ & astrocytoma & 2 & 3 & 1 & 6 \\
\hline 3 & $23 / \mathrm{M}$ & $\mathrm{L}$ & frontal & astrocytoma & 3 & 1 & 2 & 5 \\
\hline 4 & $17 / \mathrm{M}$ & $\mathrm{L}$ & temporal & astrocytoma & 3 & 1 & 2 & 8 \\
\hline 5 & $25 / \mathrm{M}$ & $\mathrm{R}$ & parietal & astrocytoma & 0 & 0 & 0 & 0 \\
\hline Total & & & & & 8 & 5 & 9 & 20 \\
\hline
\end{tabular}

$\mathrm{S}=$ Subtraction; $\mathrm{M}=$ multiplication; $\mathrm{MS}=$ multiplication + subtraction; no = no multiplication task. sites, without naming or reading interference, were found in the left hemisphere (fig. 1). No number-specific interference sites were found in the right hemisphere.

\section{Localization of Numerical Processing Sites}

Eight sites were multiplication-specific sites (no subtraction interference). Nine sites were subtraction-specific sites (no multiplication interference). Five sites were multiplication and subtraction interference sites. These results are summarized in table 1 . The maximal current ranged from 4 to $6 \mathrm{~mA}$.
Overall, numerical processing interferences were found in 3 particular regions: angular gyrus (5 interferences: 1 multiplication, 3 subtraction and 1 multiplication/subtraction); the horizontal segment of the IPS (12 interferences: 2 multiplication, 6 subtraction and 4 multiplication/subtraction), and supramarginal gyrus (5 interferences: 5 multiplication). These results are summarized in table 2 . Subcortical numerical processing stimulations were used for 1 patient, and numerical processing interferences were not found. 
Fig. 2. a CT 1 night after the operation. b MRI 1 day after the operation.
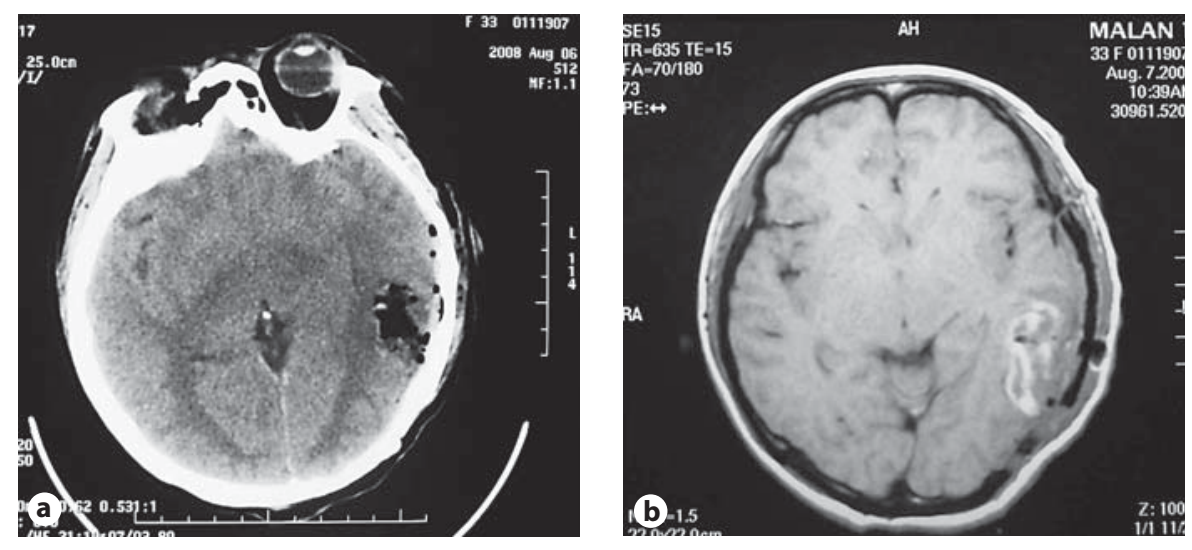

Table 2. Localization of numerical processing sites in 4 patients treated in the left hemisphere

\begin{tabular}{llll}
\hline $\begin{array}{l}\text { Patient } \\
\text { No. }\end{array}$ & Angular gyrus & HIPS & $\begin{array}{l}\text { Supramarginal } \\
\text { gyrus }\end{array}$ \\
\hline 1 & $1 \mathrm{~S}$ & $3 \mathrm{~S}$ & \\
2 & $1 \mathrm{~S}$ & $1 \mathrm{M} ; 3 \mathrm{MS}$ & $1 \mathrm{M}$ \\
3 & $1 \mathrm{MS}$ & $1 \mathrm{M} ; 2 \mathrm{~S}$ & $2 \mathrm{M}$ \\
4 & $1 \mathrm{~S} ; 1 \mathrm{M}$ & $1 \mathrm{~S} ; 1 \mathrm{MS}$ & $2 \mathrm{M}$ \\
\hline Total & $5(1 \mathrm{M} ; 3 \mathrm{~S} ; 1 \mathrm{MS})$ & $12(2 \mathrm{M} ; 6 \mathrm{~S} ; 4 \mathrm{MS})$ & $5(5 \mathrm{M})$ \\
\hline
\end{tabular}

HIPS = Horizontal segment of the IPS; $\mathrm{S}=$ subtraction; $\mathrm{M}=$ multiplication; $\mathrm{MS}=$ multiplication + subtraction.

\section{Extent of Resection}

The immediate postoperative MRI procedures showed complete tumor removals in all patients. Postoperative CT/MRI revealed gross-total resections in 4 out of 5 patients. There were no deaths. For 1 patient, the surgical site was very close to the numerical processing sites $(<1 \mathrm{~cm})$. For the other patients, numerical processing interference sites were far from the surgical site $(>1 \mathrm{~cm})$ or were spared from surgery (fig. 2).

\section{Postoperative Course}

One patient showed symptoms of right inferior quadrantanopia. Numerical processing tasks were performed in all patients. Four patients did not have any postoperative numerical processing difficulties. One patient had numerical processing difficulties 1 month after surgery, but did not have any language difficulties. The margin of the tumor removal of this patient was very close $(<1 \mathrm{~cm})$ to the numerical processing areas. This patient was tested again after 6 months of his operation. Mild numerical processing difficulties persisted. The patient was able to read and write Arabic numbers, answer single-digit multiplication questions more easily and faster than 6 months before, and solve these questions without any mistakes. He could do mental arithmetic subtraction normally, even serial subtractions, such as $100-7-7$.

\section{Discussion}

Mathematics is one of the most important and basic functions of the human brain in our daily lives [1]. Nowadays, neuroimaging and neuropsychological studies indicate that the parietal lobe, especially IPS, plays an important role in processing and representing numerical magnitude [2-4].

In previous studies, the activities of functional areas were evaluated based on blood oxygenation level-dependent functional MRI signals. Although neuroimaging and neuropsychological studies have established a frame of the role of IPS, they are not able to locate the functional areas directly and precisely. In addition, it is hard to define the exact location of brain lesions in patients with numerical processing deficits. Intraoperative electrocortical stimulation produces an electrical stream at a small part of the cortex and temporarily disturbs functioning of these areas $[5,7,8]$. Hence the functional evidence of the stimulated areas can be revealed directly, specifically and accurately. Nonetheless, only a few direct electrostimulation studies have been published so far that examine the neural basis of numerical processing $[1,9,10]$. Whalen et al. [10] were the first to use electrocortical stimulation to investigate the relationship between arithmetic problems and IPS. The results showed that stimula- 
tion at the left parietal impaired abilities to solve simple multiplication problems. Duffau et al. [9] found that the left angular gyrus was the key area for subtraction and multiplication. Roux et al. [1] found interference with 2-digit plus 2-digit addition and symptoms of Gerstmann syndrome when stimulating the angular gyrus.

In this study, our results confirmed that the essential cortices for numerical processing in multiplication and subtraction could be identified and spared from surgery. The application of brain stimulation methods allowed an accurate mapping of the region in the parietal cortices. The effects of intraoperative mapping were verified by the postoperative clinical course, that is, all the crucial numerical processing sites could be preserved. Therefore, in order to improve the quality of resection and to minimize the neurological deficits, functional boundaries of numerical processing areas should be implemented during the removal of a parietal LGG.

\section{The Role of Bilateral IPS in Mathematics}

Several brain-imaging experiments have shown significant activation of the bilateral or unilateral IPS when subjects compare the magnitude of 2 numbers, enumerate dots, or solve simple and complex numerical processing problems (addition, multiplication, subtraction and division) [14-16]. In addition, IPS activation seems to be the neural instantiation of a domain-specific system for representing and processing numerical magnitude. And it is independent of the language and the number format (Arabic or in words) [17-19].

Single-case studies show that brain-damaged patients with focal parietal lesions can exhibit striking deficits in numerical processing despite well-preserved language and semantic abilities [20,21]. The double dissociations suggest that the bilateral inferior parietal network is dedicated to the mental manipulation of numerical quantities. Recently, 2 voxel-based lesion-symptom mapping studies demonstrated that arithmetic was associated with a small number of foci and the most significant of them are located in the left inferior parietal lobule, whereas comprehension was highly associated with left middle and superior temporal gyri $[22,23]$. However, the role of the right IPS in mathematics processes is still under debate. It remains an open question whether the right IPS plays the same critical role as the left IPS in numerical processing.

Our electrical stimulation results showed that $22 \mathrm{nu}-$ merical processing-specific sites were found in the left hemisphere, and no numerical processing-specific interference sites were found in the right hemisphere. More recently, numerical processing mapping was used in several different studies $[1,9,10]$. Cortical areas on the dominant parietal lobe involved in numerical processing can be detected by using different tasks. But there were no reports on whether non-dominant parietal lobe is involved in numerical processing functions. Our results thus support the conclusion that the IPS is involved in numerical processing, as demonstrated in previous neuroimaging studies which showed bilateral IPS activations [15-17]. Moreover, our investigation specifies that only the left IPS is necessary for multiplication and subtraction processing, whereas the right IPS does not appear to be critically involved in this process. Our results are well in accordance with the recent functional imaging and transcranial magnetic stimulation studies of the involvement of the left hemisphere in number processing [23, $24]$. This is very interesting. To our knowledge, it could be the first time to find that numerical processing is originated predominantly in the left/dominant cerebral hemisphere through brain stimulation methods. That is, just like language, calculation function is found in the left hemisphere or in the dominant hemisphere. If this hypothesis is true, more attention should focus on the dominant hemisphere either in fMRI studies or in numerical processing mapping.

However, only 5 patients (4 dominant and 1 non-dominant) were studied in our research. Studies with larger samples of patients are needed to confirm this finding. The absence of deficits after brain stimulation over the right IPS does not mean that this region is not involved in numerical processing, but indicates that it is not crucial for numerical processing in the range selected for this study. Complex tasks are assumed to be processed sequentially across multiple stages including arithmetic fact retrieval, working memory and short-term memory processing. Whether complex numerical processing tasks might involve bilateral parietal lobes or not, as compared with simple tasks, is worth further investigation.

\section{Conclusion}

To improve the quality of resection while minimizing the neurological deficits, functional boundaries of numerical processing areas should be used during the removal of a parietal LGG. Moreover, only the left IPS is necessary for numerical processing, whereas the right IPS does not appear to be critically involved in this process. That is, just like language, numerical processing function has been found in the left hemisphere or in the dominant 
hemisphere. We conclude that studying the different involvement of both hemispheres and their different numerical processing styles may help to understand functions and impairments of numerical processing in the future.

\section{Acknowledgements}

This study was supported by grants from the National Key Project of Science and Technology Supporting Programs of China (No. 2007BAI05B08), and the Major State Basic Research Development Program of China (973 Program) (No. 2007CB12503).

\section{References}

1 Roux FE, Boukhatem L, Draper L, Sacko O, Démonet JF: Cortical calculation localization using electrostimulation. J Neurosurg 2009;110:1291-1299.

$\checkmark 2$ Dehaene S, Spelke E, Stanescu R, Pinel P, Tsivkin S: Sources of mathematical thinking: behavioral and brain-imaging evidence. Science 1999;284:970-974.

$\checkmark 3$ Dehaene S, Piazza M, Pinel P, Cohen L: Three parietal circuits for number processing. Cogn Neuropsychol 2003;20:487-506.

$\checkmark 4$ Andres M, Seron X, Olivier E: Hemispheric lateralization of number comparison. Cogn Brain Res 2005;25:283-290.

5 Ojemann G, Ojemann J, Lettich E, Berger M: Cortical language localization in left, dominant hemisphere: an electrical stimulation mapping investigation in 117 patients. J Neurosurg 1989;71:316-326.

-6 Ojemann JG, Miller JW, Silbergeld DL: Preserved function in brain invaded by tumor. Neurosurgery 1996;39:253-258.

7 Sanai N, Mirzadeh Z, Berger MS: Functional outcome after language mapping for glioma resection. N Engl J Med 2008;358:18-27.

$\checkmark 8$ Duffau H, Capelle L, Sichez N, Denvil D, Lopes M, Sichez JP: Intraoperative mapping of the subcortical language pathways using direct stimulations: an anatomo-functional study. Brain 2002;125:199-214.

-9 Duffau H, Denvil D, Lopes M, Gasparini F, Cohen L, Capelle L: Intraoperative mapping of the cortical areas involved in multiplication and substraction: an electrostimulation study in a patient with a left parietal glioma. J Neurol Neurosurg Psychiatry 2002;73:733736.
10 Whalen J, McClosley M, Lesser RP, Gordon B: Localizing arithmetic processes in the brain: evidence from atransient deficit during cortical stimulation. J Cogn Neurosci 1997;3:409-417.

11 Wu CX, Pu S, Lin Y, Wang YZ, Jiang T, Xie J: Fractionated resection on low grade gliomas involving Broca's area and insights to brain plasticity. Chin Med J (Engl) 2008;121:2026 2030.

-12 Xie J, Chen XZ, Jiang T, Li SW, Li ZX, Zhang $\mathrm{Z}$ : Preoperative blood oxygen level-dependent functional magnetic resonance imaging in patients with gliomas involving the motor cortical areas. Chin Med J (Engl) 2008;121:631-635

13 Zhang Z, Jiang T, Xie J, Liu FS, Li SW, Qiao $\mathrm{H}$ : Surgical strategies for glioma involving language areas. Chin Med J (Engl) 2008;121: 1800-1805.

14 Valerie D, Michael A, Mauro P: Dissociation of numerosity and duration processing in the left intraparietal sulcus: a transcranial magnetic stimulation study. Cortex 2008;44: 462-469.

15 Piazza M, Mechelli A, Price C, Butterworth B: Exact and approximate judgements of visual and auditory numerosity: an fMRI study. Brain Res 2006;1106:177-188.

16 Piazza M, Izard V, Pinel P, Lebihan D, Dehaene S: Tuning curves for approximate numerosity in the human intraparietal sulcus. Neuron 2004;44:547-555.
17 Piazza M, Mechelli A, Butterworth B, Price CJ: Are subitizing and counting implemented as separate or functionally overlapping processes? Neuroimage 2002;15:435-446.

$>18$ Venkatraman V, Ansari D, Chee M: Neural correlates of symbolic and non-symbolic arithmetic. Neuropsychologia 2004;43:744753.

19 Ashkenazi S, Henik A, Ifergane G, Shelef I: Basic numerical processing in left intraparietal sulcus (IPS) acalculia. Cortex 2008;44: 439-448.

20 Dehaene S: Varieties of numerical abilities. Cognition 1992;44:1-42.

21 Dehaene S, Cohen L: Two mental calculation systems: a case study of severe acalculia with preserved approximation. Neuropsychologia 1991;29:1045-1074.

22 Dehaene S, Dehaene-Lambertz G, Cohen L: Abstract representations of numbers in the animal and human brain. Trends Neurosci 1998;21:355-361.

23 Fehr T, Code C, Herrmann M: Auditory task presentation reveals predominantly right hemispheric fMRI activation patterns during mental calculation. Neurosci Lett 2008; 431:39-44.

24 Andre Knops, Hans-Christoph Nuerk, Roland Sparing, Henrik Foltys, Klaus Willmes: On the functional role of human parietal cortex in number processing: how gender mediates the impact of a 'virtual lesion' induced by rTMS. Neuropsychologia 2006;44: 2270-2283. 